$$
\text { CONF- } 9307124--5
$$

Note: This is a preprint of a paper being submitted for publication. Contents of this paper should not be quoted nor referred to without permission of the author(s).

\title{
Raman Study of "Boson Peak" in Ion-Implanted GaAs: \\ Dependence on Ion Dose and Dose Rate
}

\author{
M. Ivanda and U. V. Desnica \\ Ruder Boskovic Institute \\ Croatia \\ T. E. Haynes \\ Oak Ridge National Laboratory \\ Oak Ridge, Tennessee \\ "The submitted manuscript has been \\ authored by a contractor of the U.S. \\ Government under contract No. DE. \\ AC05-840R 21400. Accordingly, the \\ AC. \\ royalty-free license to publish or \\ reproduce the published form of this \\ contribution, or allow others to do so, \\ for U.S. Government purposes." \\ Prepared by the \\ Oak Ridge National Laboratory \\ Oak Ridge, Tennessee 37831 \\ managed by \\ MARTIN MARIETTA ENERGY SYSTEMS, INC \\ for the \\ U.S. DEPARTMENT OF ENERGY \\ under contract DE-AC05-84OR21400
}

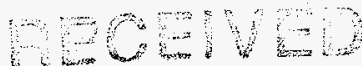

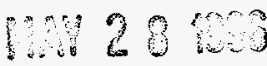

(3)

November 1993

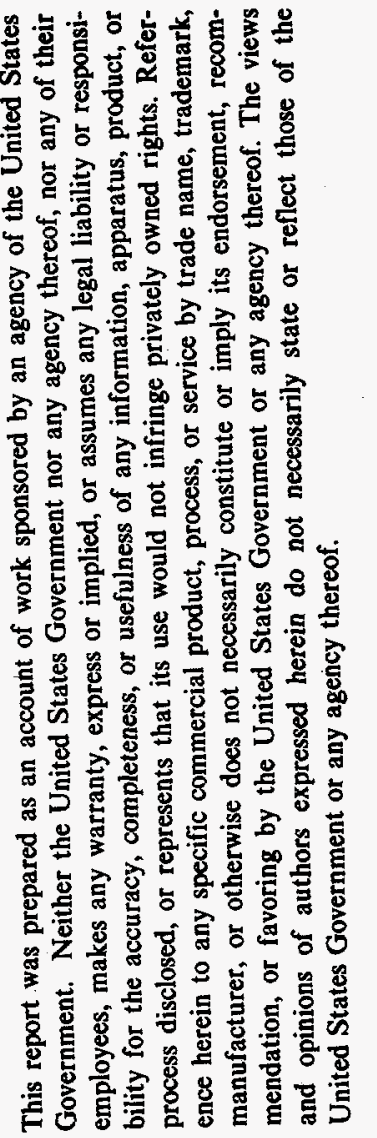




\section{DISCLAIMER}

Portions of this document may be illegible in electronic image products. Images are produced from the best available original document. 


\title{
RAMAN STUDY OF "BOSON PEAK" IN ION-IMPLANTED GaAs: DEPENDENCE ON ION DOSE AND DOSE RATE
}

\author{
M. IVANDA ${ }^{1}$, U. V. DESNICA ${ }^{1}$ AND T. E. HAYNES ${ }^{2}$

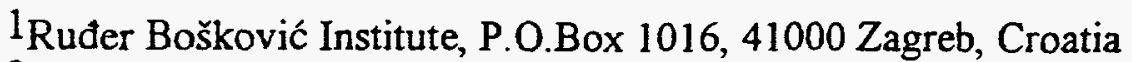 \\ 2Oak Ridge National Laboratory, P.O.Box 2008, Oak Ridge, Tennessee 37831,USA
}

Key words: Raman scattering, GaAs, Ion implantation, Fractal analysis

\begin{abstract}
Here we present a systematic Raman study of the "boson peak" which appears in GaAs during the processes of amorphization: influence of the ion doses and dose rates. The findings were interpreted in the fractal model and correlated with recent analysis of ion-induced damage from Raman and ion channeling measurements. The Raman spectra were decomposed on phonon-fracton curve and Gaussian bands by fitting procedure. The crossover frequency $\omega_{\text {col }}$ between phonon and fracton regimes and the fractal exponent $(\sigma+d-D) \tilde{d} / D$ shows a pronounced dependence on applied ion dose and weak dependence on dose rate. Evolution of the fractal component is compared with ion channeling measurements and Raman spectra of phonon bands. The fractal component is strongly dependent on ion dose as is the amorphous component and is weakly dependent on dose rate. It indicates that the fractal component is not connected with point crystalline defects, to which ion channeling is particularly sensitive. The fractal correlation length $\xi$ and spectral dimension $d$, calculated from the crossover frequency and fractal exponent, changes from $\xi=6 \AA$ and $\widetilde{d}=0.2$ for weakly damaged samples, to $\xi=10 \AA$ and $\tilde{\mathrm{d}}=0.8$ for completely amorphized samples.
\end{abstract}

\section{INTRODUCTION}

A great number of experimental results obtained recently show that many properties (excitation and relaxation kinetics, thermal, optical and electron processes) of disordered solids depend on structural features at length scale from atomic level to $100 \AA$. Low-frequency vibrational spectroscopy is an efficient method for investigation of structural properties on such a scale, often called a scale of medium range order. It seems that structural properties at medium range order cause the excess of the vibrational density of states in comparison to the expected Debye value. The so-called "boson peak" at low energies in the Raman scattering spectra is a manifestation of such "excess" modes. The "boson peak" has been observed in vitreous semiconductors like a-Se, a$\mathrm{As}_{2} \mathrm{Se}_{3}$, but in tetrahedral like a-Si has not been found and, therefore, was often identified with TA phonon vibrational band [4]. Recently, the true "boson peak" has been discovered in a-Si:H [1] and a-GaAs [2]. Also, it has been shown that the "boson peak" can be successfully explained by applying the fractal model [3]. Here, we present a systematic Raman study of the "boson peak" in GaAs which develops during the processes of amorphization. The evolution of the "boson peak" for different ion doses and dose rates (current densities) is presented. 


\section{RESULTS AND DISCUSSION}

Recently, the theory for the Raman scattering from fractons has been developed [6]. The calculation was based on dipole-induceddipole (DID) mechanism that has becn recognized as the major source of Raman scattering in dense systems. We have shown that the origin of the "boson peak" can be successfully explained by applying the fractal model. The phonon-fracton curve of the temperature reduced Raman intensity, $I^{R}(\omega)$, is given by [3]:

$$
I^{R}(\omega) \propto \omega^{3}\left(\omega^{2}+\omega_{\infty 1)}^{2}\right)^{\tilde{\mid}|\vec{d} D(\sigma+d-D)-5 / 2|}
$$

where $\omega_{\text {col }}$ is the crossover frequency from phonon to fracton scattering regime, $\tilde{\mathrm{d}}$ the spectral dimension, D fractal dimension, $d$ space dimension and $\sigma$ the scaling index describing the modulation of the density in the embedding space by the vibration. Fig. 2 shows the Raman spectra decomposed on the phonon-fracton curve and the vibrational bands. It is evident that the intensity of the "boson peak" dramatically increases with ion dose. Also, as a result of decomposition spectra, the fracton exponent $(\sigma+d-D) \tilde{d} / D$ increases (fig. 3a) and the crossover frequency $\omega_{\mathrm{col}}$ decreases (fig. $3 \mathrm{~b}$ ) with ion dose, while the dependence on current density is much weaker (open squares in fig.3). Recent comparison of the Raman spectra of the phonon bands with the ion channeling measurements shows that the damage morphology in GaAs is dependent upon both dose and dose rate, and that the dose-ratedependent component of the total damage consists primarily of crystalline defects [5]. Our results (obtained on the same set of the samples) lead to the same conclusion. The fractal component is strongly dependent on ion dose as the amorphous one and is weakly dependent on dose rate. It indicates that the fractal component is not connected with point crystalline defects, to which ion channeling is particularly sensitive.

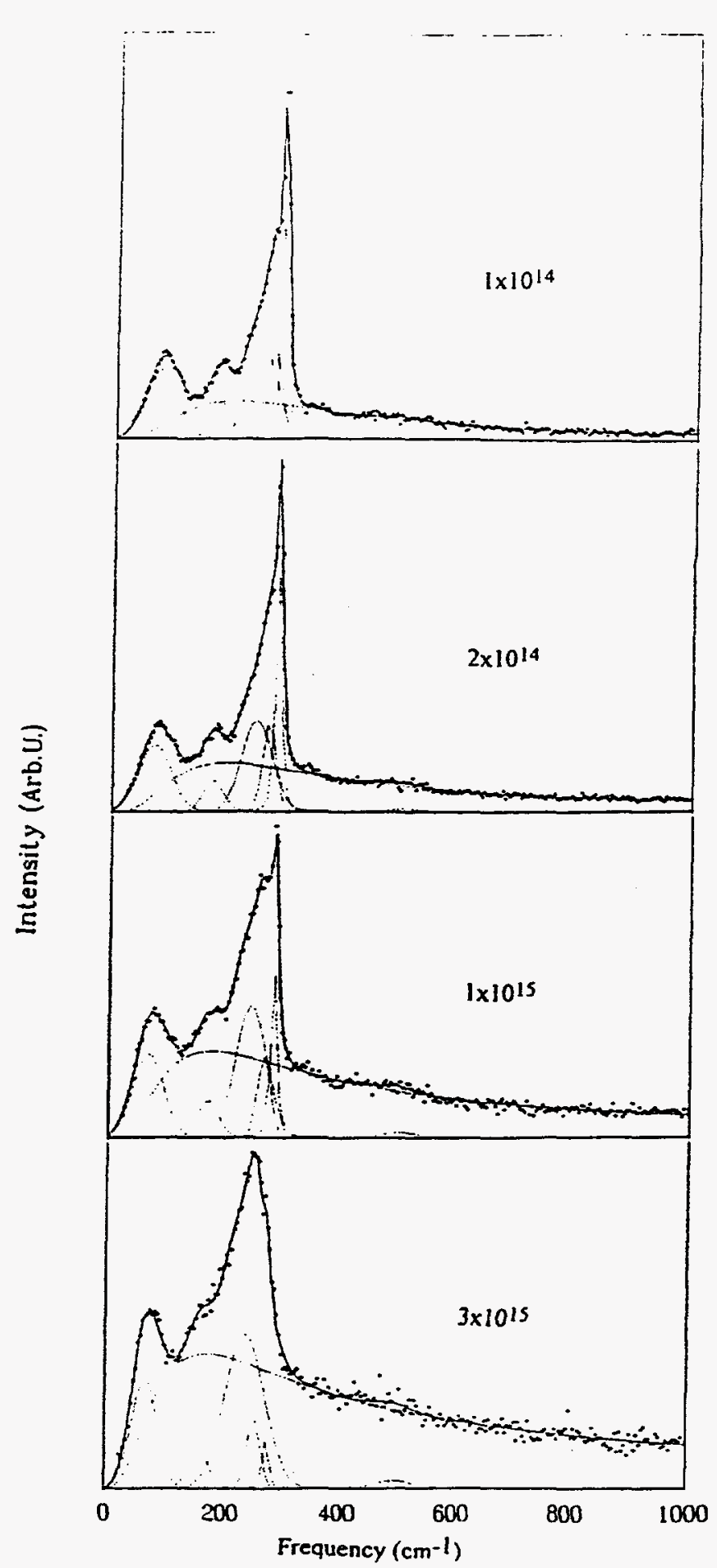

Figure 2. Raman spectra of $100-\mathrm{keV}{ }^{30} \mathrm{Si}^{+}$implanted GaAs decomposed on the vibrational bands and phonon-fracton curve. Implantation current was fixed at $0.05 \mu$ $\mathrm{A} / \mathrm{cm}^{2}$; fluences in ions $/ \mathrm{cm}^{2}$ are indicated on the figure. 